\title{
Properties and Stability of Ge-As-Se Evaporated Thin Films for Nonlinear Waveguides
}

\author{
Barry Luther-Davies, Douglas Bulla, Rongping Wang, Amrita Prasad, Steve Madden, Duk-Yong Choi \\ Centre for Ultra-High-Bandwidth Devices for Optical Systems, Laser Physics Centre, RSPE, The Australian National University, Canberra \\ ACT0200, Australia
}

Chalcogenide glasses are an important class or amorphous materials with applications that include phasechange optical memories, solar cells, and optical waveguides. Particularly interesting for optical applications is their good transparency in the mid-infrared combined with their high linear and nonlinear refractive indices. This last property has motivated interest in the use of chalcogenide waveguides for all-optical processing of highspeed telecommunications signals [1].

For many of these applications an essential step in device fabrication is the preparation of high quality optical thin films free from defects and with stable physical properties. It is well known that chemical bonds within chalcogenide glasses can relax under exposure to light, heat, electron bombardment, etc. This results in changes to the physical properties that causes undesirable drifts in the device properties. The chemical bonds in evaporated thin films often differ significantly from those of the bulk making films even less stable.

In this work we posed some simple questions, namely, how do the deposition conditions during thermal evaporation affect film properties, and how do those properties and particularly their stability vary with the glass composition? To elucidate this we studied a series of glasses from the Ge-As-Se system which has one of the broadest glass-forming regions of any of the chalcogenides. We prepared bulk glasses by a standard meltquenching approach, and thin films by thermal evaporation in a baffled boat in high vacuum. A wide range of material parameters were measured: bond structure was probed by x-ray photoelectron spectroscopy and Raman spectroscopy; bulk glass properties such as $\mathrm{T}_{\mathrm{g}}$, density, refractive index, Kerr nonlinearity, band gap, and elastic properties were also measured. The properties of as-deposited films were compared with those of the equivalent bulk glass and the effect of annealing on those properties was carefully characterized.

A number of important conclusions emerged from this work. Firstly it was found that many physical properties varied as a function of mean coordination number, $\mathrm{MCN}$ (the sum of the products of the individual atomic valencies times their atomic weight percent). It has been predicted that distinct changes in glass structure occur at $\mathrm{MCN} \approx 2.4$ and $\mathrm{MCN} \approx 2.67$ due to transitions from floppy to rigid glass network and from 2-D rigid to 3$\mathrm{D}$ stressed rigid state $[2,3]$. These transitions were apparent in the MCN-dependence of density, elastic constants, refractive index, band-gap etc. Thus we found that network structure was a major factor determining glass properties in this ternary system.

From the device point of view, however, the important finding was that the differences in physical properties between thermally deposited films and bulk glasses disappeared over a small range of MCN (2.4-2.55) - see for example Fig. 1. We found that in this small range of MCN, the glass state, which represents the global minimum of the potential energy of the network, is not separated by potential barriers from other amorphous states. Hence films in this range of MCN form directly in the glass state. These particular films were also much more stable compared with those with higher or lower MCN. We, therefore, concluded that it is possible to identify stable films with high nonlinearity and are now using these glasses for photonic devices.

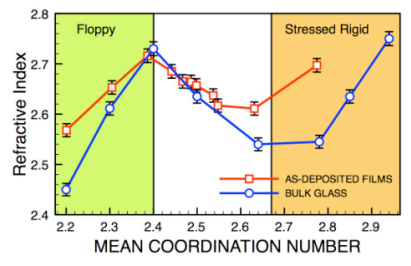

Fig. 1 Variation of refractive index of Ge-As-Se glasses as a function of MCN for both films and bulk glass showing the range of $\mathrm{MCN}$ for which films were indistinguishable from the bulk.

\section{References}

[1] M. Pelusi, V. Ta’eed, E. Magi, M. Lamont, S. Madden, Duk-Yong Choi, D. Bulla, B. Luther-Davies, B. Eggleton, “Applications of higlynonlinear chalcogenide glass devices tailored for high-speed all-optical signal processing”. IEEE Sel. Topic in Quantum Electr. $14,529-539$. (2008); M.1 Galili, J. Xu, H. Mulvard, L. Oxenlöwe, A. Clausen, P. Jeppesen, B. Luther-Davies, S. Madden, A. Rode, Duk-Yong Choi, M. Pelusi, F. Luan, B. Eggleton, Breakthrough switching speed with and all optical chalcogenide glass chip: 640Gb.s demultiplexing" Optics Express (2008) in press.

[2] J. C. Phillips, “Topology of covalent non-crystalline solids I: Short-range order in chalcogenide alloys”, J. Non-Cryst. Solids, 34, No. 2, 153 (1979) ; P. Boolchand, D. G. Georgiev and B. Goodman, "Discovery of the intermediate phase in chalcogenide glasses", J. Optoelectron. Adv. Mat., 3, no.3, 703 (2001) ; M. F. Thorpe, D. J. Jacobs, M.V. Chubynsky and J. C. Phillips, "Self organization in glass networks", J. Non-Cryst. Solids 266-269, 859 (2000).

[3] K. Tanaka, "Structural phase transitions in Chalcogenide glasses", Phys. Rev. B, 39, No. 2, 1270 (1989). 